\title{
METAL-FERROELECTRIC-SEMICONDUCTOR FIELD-EFFECT TRANSISTOR NAND GATE SWITCHING TIME ANALYSIS
}

\author{
THOMAS A. PHILLIPS ${ }^{\mathrm{a}}$, TODD C. MACLEOD ${ }^{\mathrm{a}}$, and FAT D. HO ${ }^{\mathrm{b} *}$ \\ ${ }^{a}$ National Aeronautics and Space Administration, Marshall Space Flight Center, \\ Huntsville, Alabama, 35812, U.S.A. \\ ${ }^{b}$ The University of Alabama in Huntsville, Department of Electrical and Computer Engineering, \\ Huntsville, Alabama 35899, U.S.A.
}

\begin{abstract}
Previous research investigated the modeling of a NAND gate constructed of Metal-FerroelectricSemiconductor Field-Effect Transistors (MFSFETs) to obtain voltage transfer curves. The NAND gate was modeled using n-channel MFSFETs with positive polarization for the standard CMOS n-channel transistors and n-channel MFSFETs with negative polarization for the standard CMOS p-channel transistors. This paper investigates the MFSFET NAND gate switching time propagation delay, which is one of the other important parameters required to characterize the performance of a logic gate. Initially, the switching time of an inverter circuit was analyzed. The low-to-high and high-to-low propagation time delays were calculated. During the low-tohigh transition, the negatively polarized transistor pulls up the output voltage, and during the high-to-low transition, the positively polarized transistor pulls down the output voltage. The MFSFETs were simulated by using a previously developed model which utilized a partitioned ferroelectric layer. Then the switching time of a 2-input NAND gate was analyzed similarly to the inverter gate. Extension of this technique to more complicated logic gates using MFSFETs will be studied.
\end{abstract}

* Author to whom correspondence should be addressed. 


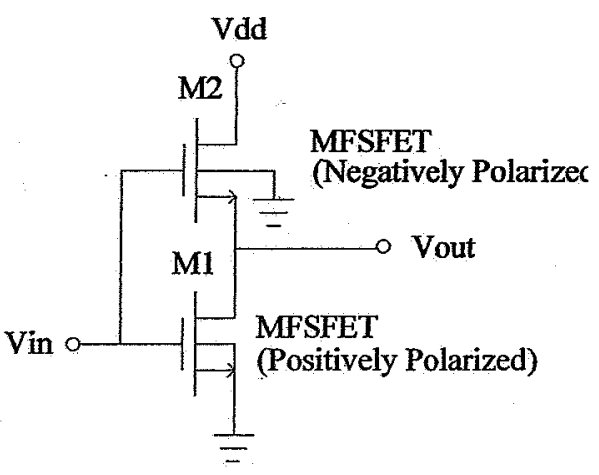

Figure 1: MFSFET Inverter Circuit

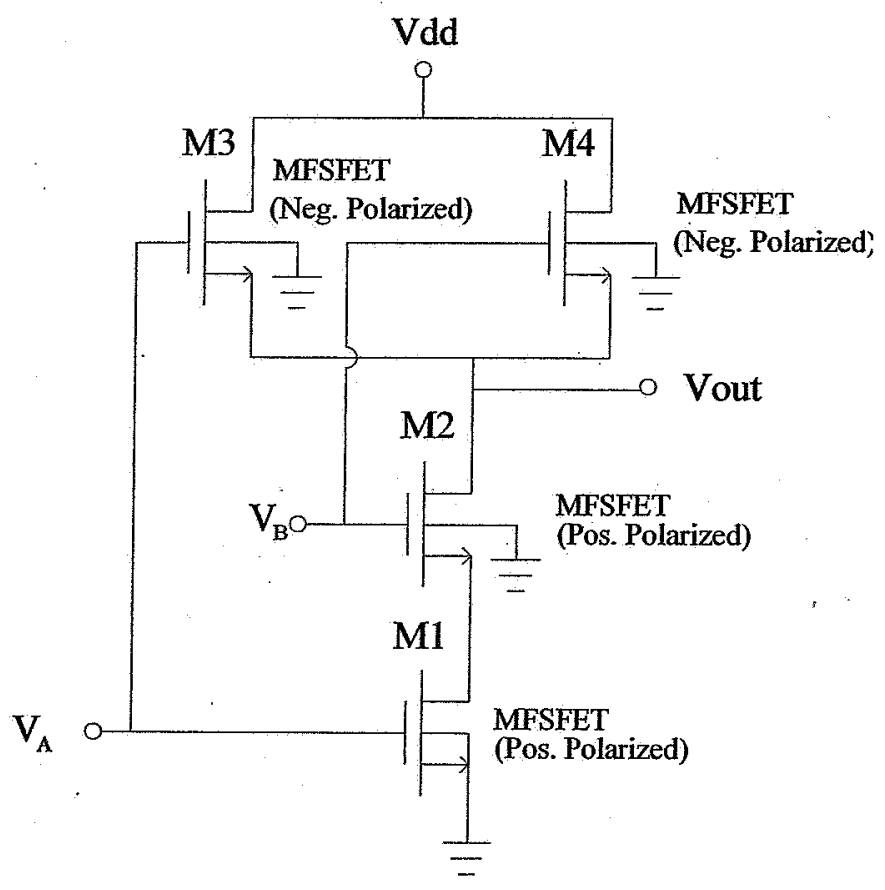

Figure 2: 2-Input MFSFET NAND Gate 